This document is the accepted manuscript version of the following article: Wang, X., Qin, X. H., Hu, C., Terzopoulou, A., Chen, X. Z., Huang, T. Y., ManiuraWeber, K., Pané, S., \& Nelson, B. J. (2018). 3D printed enzymatically biodegradable soft helical microswimmers. Advanced Functional Materials, 28(45), 1804107 (8pp.). https://doi.org/10.1002/adfm. 201804107

\title{
3D Printed Enzymatically Biodegradable Soft Helical Microswimmers
}

Xiaopu Wang, Xiao-Hua Qin*, Chengzhi Hu, Anastasia Terzopoulou, Xiang-Zhong Chen, Tian-Yun Huang, Katharina Maniura-Weber, Salvador Pané* and Bradley J. Nelson

X. Wang, Dr. C. Hu, A. Terzopoulou, Dr. X.-Z. Chen, Dr. T.-Y. Huang, Dr. S. Pané, Prof. B. J. Nelson Multi-Scale Robotics Lab

Institute of Robotics and Intelligent Systems

ETH Zurich ,Tannenstrasse 3

CH-8092 Zurich, Switzerland

E-mail: vidalp@ethz.ch

Dr. X.-H. Qin, Prof. K. Maniura-Weber

Biointerfaces Lab

Empa, Swiss Federal Laboratories for Materials Science and Technology

CH-9014 St. Gallen, Switzerland

E-mail: xh.qin84@gmail.com

Current address (X.-H. Q.): Institute for Biomechanics, ETH Zurich, Leopold-Ruzicka-Weg 4, 8093 Zurich

Keywords: biodegradable hydrogel, two-photon polymerization, soft helical microswimmers, magnetic manipulation

\section{Abstract:}

Mobile micro- and nanorobots have been proposed for future biomedical applications, such as diagnostics and targeted delivery. For their translation to clinical practice, biocompatibility and biodegradability of micro- and nanorobots are required aspects. The fabrication of smallscale robots with non-cytotoxic biodegradable soft components will allow for enhanced device assimilation, optimal tissue interaction and minimized immune reactions. We report the 3D microfabrication of biodegradable soft helical microswimmers via two-photon polymerization of the nontoxic photocrosslinkable hydrogel gelatin methacryloyl (GelMA). GelMA microswimmers are fabricated with user-defined geometry and rendered magnetically responsive by decorating their surface with magnetic nanoparticles. In contrast to previous rigid helical microrobots, our soft helical microswimmers can corkscrew above the step-out frequency with relatively high values of forward velocity, suggesting an unprecedented selfadaptive behavior. Cytotoxicity assays show the toxicity of GelMA is at least three orders of 
magnitude lower than that of poly(ethyleneglycol) diacrylates, which are widely used for fabricating hydrogel-based microswimmers. GelMA microswimmers are fully degradable by collagenases. Furthermore, they support cell attachment and growth, and can be gradually digested by cell-released enzymes during culture. These non-cytotoxic biodegradable hydrogel microswimmers will greatly expand their applications in medicine by eliminating the concerns of retrieving microrobots after fulfilling tasks in body.

\section{Introduction}

Untethered mobile micro- and nanoscale robots will represent a new paradigm shift in the field of biomedical robotics in the near future. ${ }^{[1]}$ These small devices, capable of transforming different types of energy into locomotion, have demonstrated great potential in targeted drug delivery, ${ }^{[2]}$ microsurgery, ${ }^{[3]}$ sensing and diagnosis. ${ }^{[4]}$ Recently, different types of small-scale robots have been evaluated in vivo, ${ }^{[5]}$ especially to investigate their motion capabilities. However, many aspects must be addressed before translating these devices to the operating theatre. A big challenge in biomedical small-scale robotics is to design functional micro- and nanoswimmers capable of realizing multiple tasks, and simultaneously fulfilling all the requirements for being implanted in the human body. ${ }^{[6]}$ Current trends in micro- and nanorobotics are towards the adoption of soft materials, ${ }^{[7]}$ which are better suited for biomedical applications, as their physicochemical properties are more similar to those of tissues. Additionally, many of them can change their physical or chemical properties upon external stimulus such as heat, mechanical stress, concentration of certain chemical species, light, ultrasound, and magnetic and electric fields, which can be advantageous for changing the locomotion mechanism or enhancing the release of therapeutic payloads. ${ }^{[7 a, 7 b]}$

While many synthetic polymers have been used for the fabrication of microrobots, ${ }^{[7 b, 8]}$ most are nondegradable despite their biocompatibility, or upon degradation they generate cytotoxic products that are difficult to be excreted from the human body. Nondegradable 
forms may also contain functional groups that elicit irritant effects to cells. Unless the microdevices are excreted or metabolized, the materials may accumulate in organs and tissues such as the liver or the adipose tissue, and trigger chronic inflammatory responses. For example, poly(N-isopropylacrylamide) (pNIPAAm) is a thermally responsive material widely used in the fabrication of microrobots. ${ }^{[7 b]}$ However, pNIPAAm is not biodegradable and, as a result, often requires copolymerization with other monomers to facilitate its degradation.

Currently, the community of small-scale roboticists is increasingly making efforts to develop biodegradable micro- and nanorobots. ${ }^{[9]}$ Wang and coworkers have recently reported transient chemically powered $\mathrm{Mg}$ - and $\mathrm{ZnO}$-based micromachines that dissolve into noncytotoxic products. ${ }^{[10]}$ Wilson's group recently reported on REDOX-sensitive stomatocyte nanomotors made of block copolymers incoporating disulfide crosslinks. The structures were successfully degraded using glutathione, a tripeptide that cleaves the disulfide groups by reduction. ${ }^{[11]}$ Peters et al have fabricated magnetic composite-based helical microswimmers by two-photon polymerization (2PP) of low molecular weight acrylates, including poly(ethylene glycol) diacrylate (PEGDA), and pentaerythritol triacrylate (PETA). Although the materials were proved degradable in alkaline conditions, ${ }^{[8]}$ these materials suffer from a few limitations. Previous studies have evidenced substantial cytotoxicity of acrylated monomers, ${ }^{[12]}$ presumably due to the high reactivity of acrylates with cell-surface proteins via the Michael addition reaction. Moreover, polyacrylic acids resulting from degradation are difficult to be excreted from the human body. ${ }^{[12 a]}$ As biodegradation within the extracellular matrix is achieved by cell-secreted proteases, ${ }^{[13]}$ the use of non-cytotoxic, biodegradable protein-based materials for fabricating robotic micro- and nanovehicles is timely.

In this paper, we report on the development of magnetically driven transient soft microswimmers made of a biodegradable protein-based photoresist. The microdevices are helical structures fabricated by means of 2PP of gelatin methacryloyl (GelMA), which is surface-decorated with magnetite nanoparticles for magnetic actuation. Because of its high 
cytocompatibility and bioactivity, GelMA is a versatile photocrosslinkable hydrogel widely employed in the fields of drug delivery and tissue engineering. ${ }^{[14]}$ GelMA is often used as a cell-laden scaffold for 3D cell culture applications as it supports cell functions such as adhesion, migration, proliferation, and differentiation. ${ }^{[12 b, 14]}$ Furthermore, GelMA hydrogels are proteolytically degradable by cell-secreted proteases in the extracellular matrix. ${ }^{[13]}$ Here, we investigate the process parameters to manufacture 3D printed GelMA helical microstructures with different size and shapes and also systematically assess the biocompatibility, biodegradability and locomotion behavior of 3D printed GelMA microswimmers in biologically relevant environments. Our cell culture experiments reveal that our microswimmers can serve as dynamic interfaces that support cell adhesion, and growth. Finally, we show that the GelMA microswimmers can be gradually digested by cellsecreted proteases in several cell cultures. The polymerization and enzymatic degradation of GelMA is illustrated in Scheme 1.
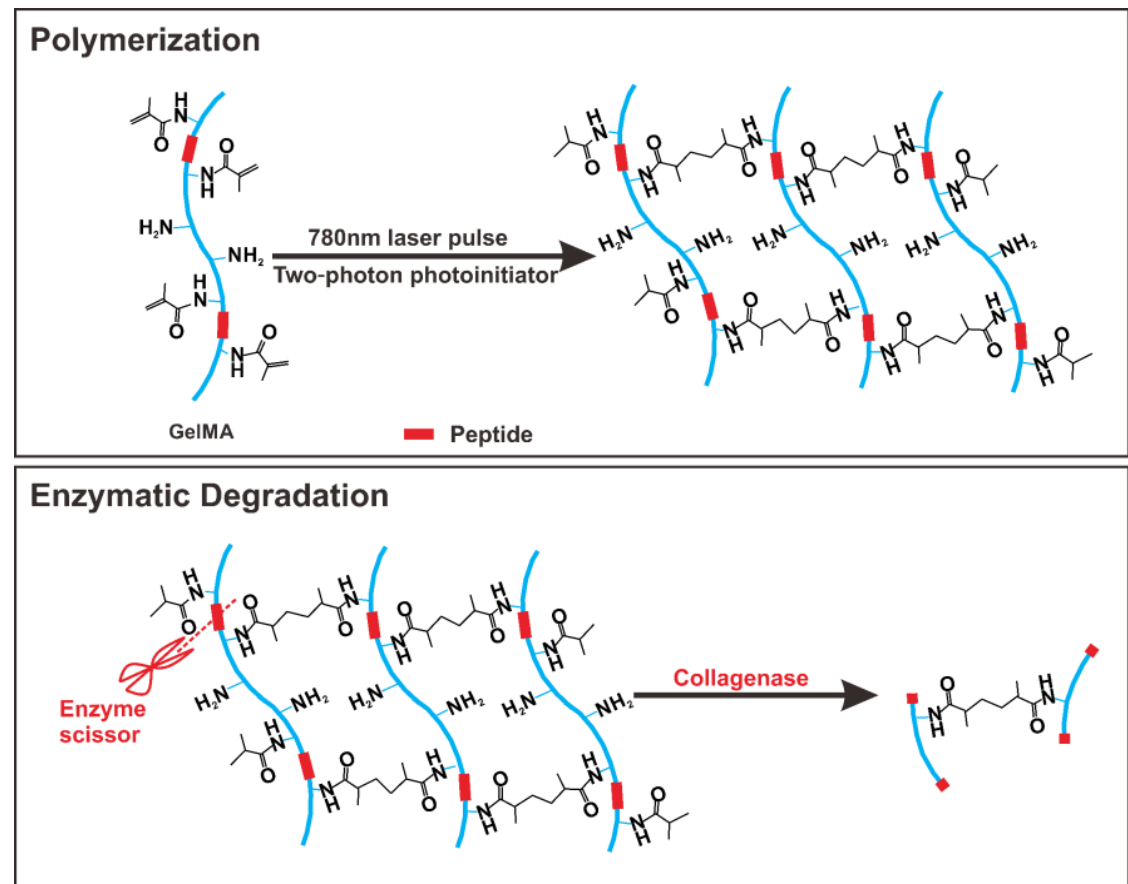

Scheme 1. Illustration of polymerization and enzymatic degradation of GelMA

\section{Results and discussion}




\subsection{Materials and fabrication}

In this study, we have chosen to 3D print GelMA microswimmers with a helical shape, as it is a widely investigated microrobotic design in terms of locomotion. ${ }^{[15]}$ To enable the twophoton polymerization of GelMA, sodium 3,39-((((1E,19E)-(2-oxocyclopentane-1,3diylidene) bis (methanylylidene))bis(4,1-phenylene))bis(methylazanediyl)) dipropanoate (P2CK) was used as a water-soluble photoinitiator. P2CK exhibits a large two-photon crosssection $(176 \mathrm{GM}$ at $800 \mathrm{~nm}) .{ }^{[16]}$ Its high efficiency in generating free radicals has previously enabled 2PP of microstructured hydrogels at high scanning speeds. ${ }^{[17]}$ The polymerization occurs via radical polymerization through the methacryloyl moieties. ${ }^{[18]}$ The chemical structures of GelMA and P2CK are shown in Scheme 2.

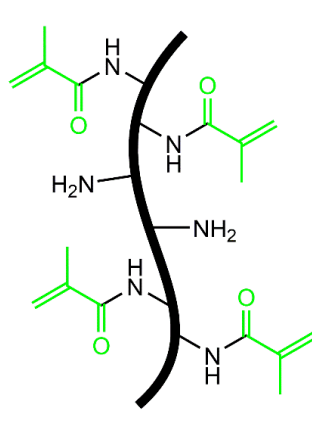

GeIMA

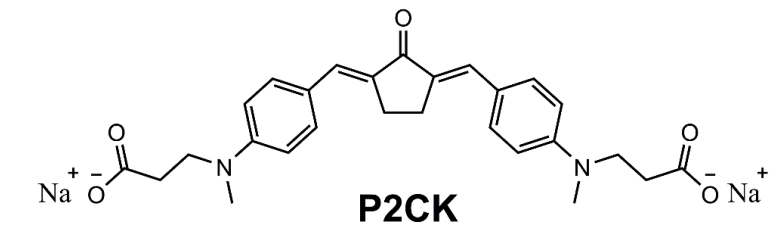<smiles>C=CC(=O)OCCOC(=O)C=C</smiles>

PEGDA (700 Da)<smiles>CC(C)(O)C(=O)c1ccc(OCCO)cc1</smiles>

Irgacure 2959

Scheme 2. Chemicals used in this work

The precursor solution was prepared by mixing GelMA (200 mg mL $\left.\mathrm{m}^{-1}\right)$ and P2CK (1 $\left.\mathrm{mg} \mathrm{mL} \mathrm{m}^{-1}\right)$ in PBS $(\mathrm{pH}=7.4)$, and subsequently polymerized upon laser irradiation at $780 \mathrm{~nm}$ wavelength. The fabrication process of magnetic helical microswimmers was carried out in two steps, as shown in Figure 1a, 1b. First, helical microstructures were printed with 2PP of GelMA. Next, these microstructures were rendered magnetic by incubating them in aqueous suspensions of magnetite $\left(\mathrm{Fe}_{3} \mathrm{O}_{4}\right)$ nanoparticles $\left(10 \mathrm{mg} \mathrm{mL}^{-1}\right)$. To evaluate the photocurability of the precursor, various laser powers and scanning speeds were evaluated. As shown in Figure 1c, 1d and 1e, helical microstructures can be fabricated in a broad processing window 
with varying laser powers and scanning speeds. As the laser power decreases or the scanning speed increases, the crosslinking density decreases, thereby compromising the stability of the helical microstructures. Based on these results, a laser power of $95 \mathrm{~mW}$ and a scanning speed of $10 \mathrm{~mm} \mathrm{~s}^{-1}$ were chosen as the optimal parameters for the fabrication of soft helical microswimmers. While the helical microswimmers fabricated with these parameters are quite soft, the structures exhibit sufficient crosslinking density and structural stability. Additionally, the size and shape of the helical microstructures is tunable due to the nature of the $2 \mathrm{PP}$ approach. Figure 1f, 1 g show helical microstructures with different sizes and different cylindrical cross-sections, respectively. Figure $\mathbf{1 h}$ shows helical microswimmers decorated with magnetic nanoparticles obtained by immersing the microstructures in a water suspension of $\mathrm{Fe}_{3} \mathrm{O}_{4}$ nanoparticles $\left(10 \mathrm{mg} \mathrm{mL}^{-1}\right.$, coated with $1 \% \mathrm{PVP}$ ) for $1 \mathrm{~h}$. The cryo-SEM image and the corresponding EDX elemental mapping for iron (Figure 1i) of GelMA helical microswimmers in their hydrated form show that the nanoparticles are uniformly distributed on the surface of the helical microstructures. Previous studies have shown the irreversible adsorption of gelatin onto the surface of mica, silica and iron oxide particles. ${ }^{[19]}$ A few research groups have recently exploited gelatin-coated iron oxide nanoparticles as stable carriers for drug delivery. ${ }^{[19 \mathrm{~d}]}$ Note that the iron oxide nanoparticles used in this paper are coated with polyvinylpyrrolidone (PVP) to prevent particle agglomeration in solution. PVP and gelatin are miscible due to the strong hydrogen bonds established between these two materials. ${ }^{[20]}$ Hence, it is hypothesized that a complex interplay of molecular interactions (hydrogen bonds, electrostatic interactions, hydrophobic interactions and van-der-Waals) are the driving forces for nanoparticle adsorption on GelMA surfaces. This approach for functionalizing GelMA microswimmers is facile and can be extended to other functional particles, drugs or sensing reagents. We also investigated another procedure to prepare magnetically responsive structures, which consisted of photocuring a mixture of GelMA, 
$\mathrm{P} 2 \mathrm{CK}$ and $\mathrm{Fe}_{3} \mathrm{O}_{4}$ nanoparticles. Detailed information can be found in supporting information

(Figure S1).

a

b
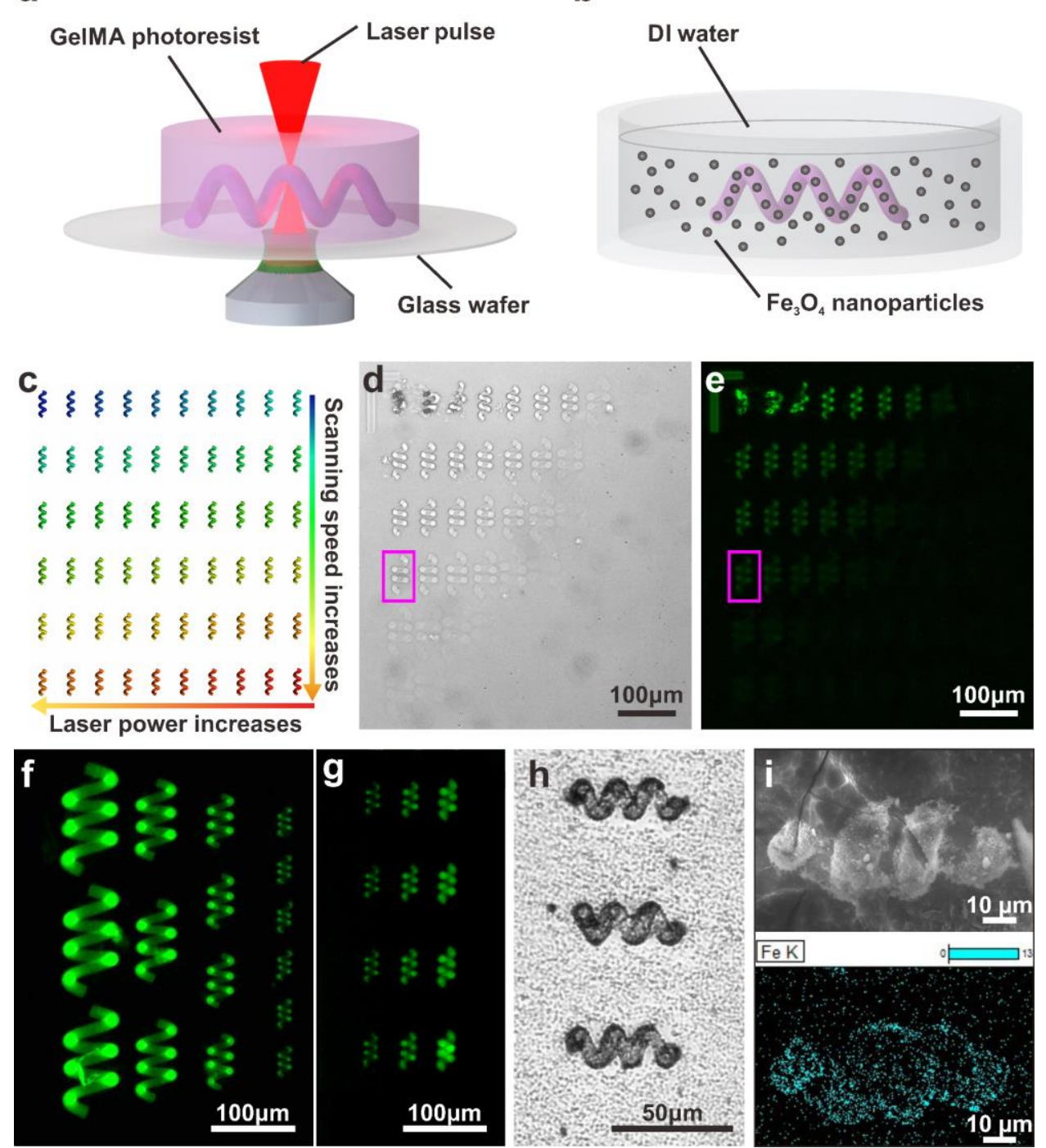

Figure 1. Schematic of the fabrication of biodegradable GelMA helical microswimmers: (a) 2PP is used to print GelMA helical microstructures; (b) the printed structures are decorated with magnetic nanoparticles by incubation in water suspensions of $\mathrm{Fe}_{3} \mathrm{O}_{4}$ nanoparticles. (c) Color chart representing microstructures fabricated with varying laser powers (from $95 \mathrm{~mW}$ to $9.5 \mathrm{~mW}$ with an interval of $9.5 \mathrm{~mW})$ and varying scanning speeds $\left(1 \mathrm{~mm} \mathrm{~s}^{-1}, 3 \mathrm{~mm} \mathrm{~s}^{-1}, 5\right.$ $\mathrm{mm} \mathrm{s}^{-1}, 10 \mathrm{~mm} \mathrm{~s}^{-1}, 30 \mathrm{~mm} \mathrm{~s}^{-1}$ and $50 \mathrm{~mm} \mathrm{~s}^{-1}$ ). (d) Optical and (e) fluorescent image of helical microstructures fabricated with varying laser powers and scanning speeds (as described in (c), 
the marked helices are those obtained using the optimal parameters. The hydrogel structures are inherently fluorescent due to residual photoinitiators. (f) Fluorescent image of helical microstructures with different sizes. (g) Fluorescent image of helical microstructures with different thicknesses. (h) Optical image of helical microswimmers decorated with magnetic nanoparticles. (i) Cryo-SEM(up) and EDX(down) mapping of iron corresponding to a GelMA microswimmer in its hydrated form.

\subsection{Swimming performance}

The helical microstructures were incubated in a $\mathrm{Fe}_{3} \mathrm{O}_{4}$ nanoparticle-water suspension $(10 \mathrm{mg}$ $\mathrm{mL}^{-1}$ ) for 1 day to provide magnetism for the swimming test. Optical images of a microswimmer made with a 1 day incubation can be found in Figure 2a. These microswimmers appear darker than those with $1 \mathrm{~h}$ incubation (Figure 1h), indicating that for a longer incubation step, more $\mathrm{Fe}_{3} \mathrm{O}_{4}$ nanoparticles are adsorbed onto the GelMA microswimmers. A rotating magnetic field (Figure 2a) of $8 \mathrm{mT}$ was applied to control swimming. The velocities of the microswimmers are schematically illustrated. Figure 2b shows the swimming velocities as a function of the rotating frequency of the applied magnetic field. The corresponding movie is provided in the supporting information (Video S1). When applying the magnetic field at a low rotating frequency $(<6 \mathrm{~Hz})$, the microswimmer showed a wobbling motion. The drift velocity was quite high and the forward velocity was relatively low, due to the interaction between the microswimmers and the substrate. As the rotating frequency of the magnetic field increased, the forward velocity increased significantly and the drifting effect lessened. Swimming then stabilized into the corkscrew region ${ }^{[21]}$ with an increase of the frequency. The microswimmer reached its step-out frequency at $16 \mathrm{~Hz}$, when the forward velocity reached its maximum. After the step-out frequency, the forward velocity of the swimming remained relatively high as the rotating frequency continuously increased. Meanwhile, the drift velocity increased. Thus, the total velocity slightly increased even after 
exceeding the step-out frequency. This is a unique swimming behavior compared to rigid helical microswimmers, whose swimming speed decreases dramatically after the step-out frequency. This unique behavior is most probably caused caused by the soft nature of the GelMA helical microswimmers. To characterize the mechanical softness of polymerized GelMA, the storage modulus of GelMA during photocrosslinking was measured using photorheology. As shown in Figure 2c, the storage modulus of photopolymerized GelMA is in the range of $40 \mathrm{kPa} \sim 50 \mathrm{kPa}$, which is much lower than SU8 photoresists ( 2 GPa), a material commonly used to fabricate rigid helical microswimmers. ${ }^{[22]}$ The low storage modulus of GelMA indicates that the microstructures are very soft and prone to tighten when they are subject to strong magnetic torques. The deformation of the helices at higher magnetic frequencies implies a decrease of the drag coefficient at high frequencies. As the drag force acting on the helical structure is always counterbalanced with the torque, an increase in the forward velocity is expected. This phenomenon suggests that our soft helical microswimmers may exhibit a self-adaptive behavior at high magnetic frequencies.
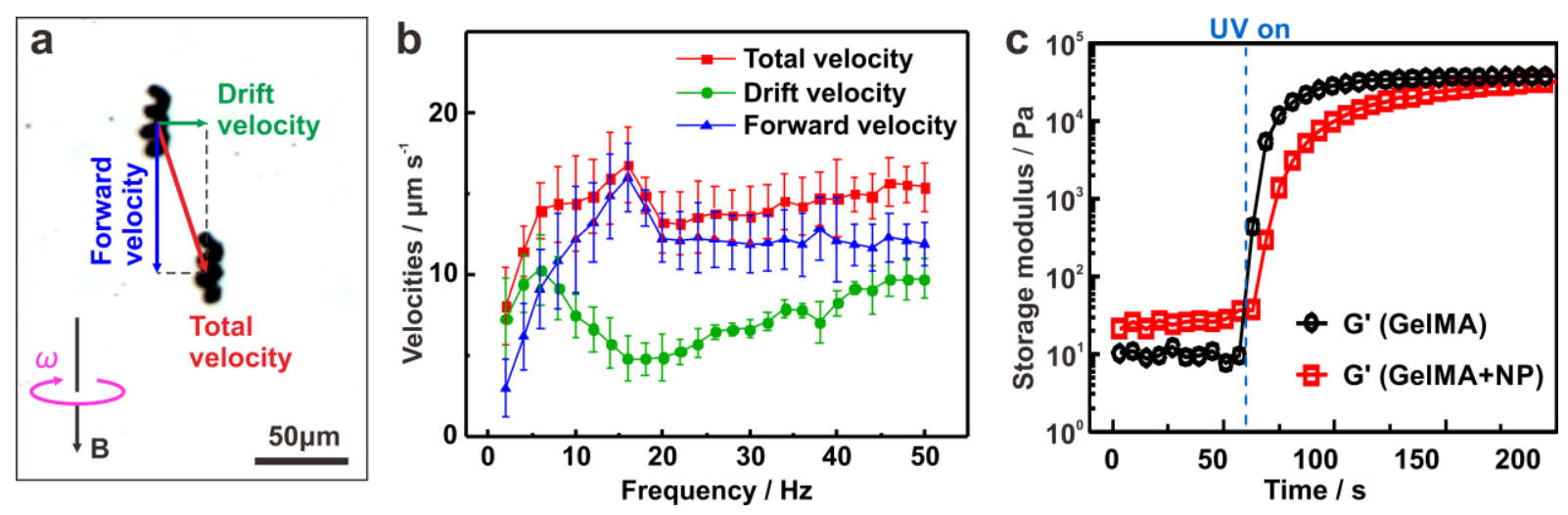

Figure 2. Swimming characterization of GelMA helical microswimmers. (a) Screenshots of a video showing a GelMA helical microswimmer moving in a biologically relevant solution (38 $\mathrm{mg} \mathrm{mL} \mathrm{m}^{-1}$ bovine serum albumin and $0.9 \mathrm{mg} \mathrm{mL}^{-1}$ glucose in PBS, $\mathrm{pH}=7.4$ ). (b) Swimming velocities of helical microswimmers as a function of rotational frequency at an applied magnetic rotating field of $8 \mathrm{mT}$; each data set corresponds to mean $\pm \mathrm{SD}(\mathrm{n}=4)$. (c) Timeresolved storage modulus (G') of GelMA formulations during UV-induced photocrosslinking 
with or without $\mathrm{Fe}_{3} \mathrm{O}_{4}$ magnetic nanoparticles measured by in situ photorheology: $20 \%$ GelMA in Irgacure 2959, UV-365 nm, $20 \mathrm{~mW} \mathrm{~cm}^{-2}$. 'GelMA+NP' represents the GelMA formulation containing $\mathrm{Fe}_{3} \mathrm{O}_{4}$ magnetic nanoparticles.

\subsection{Biocompatibility and biodegradability}

The biocompatibility of the GelMA precursor, the polymerized helical structures, and the residue of the magnetic helical microswimmers after degradation, were all evaluated in this work. The biocompatibility of GelMA precursor was systematically assessed by a 3-(4,5dimethylthiazol-2-yl)-2,5-diphenyltetrazolium bromide (MTT) assay and a lactate dehydrogenase (LDH) cytotoxicity assay. PEGDA (700 Da), the most often used photoresists for $2 \mathrm{PP}$ of hydrogels, was selected as a reference. Its chemical structure is shown in Scheme 2. Since both PEG and gelatin molecules are biocompatible materials, the toxicity analysis must focus on the influence of terminal groups: acrylates vs methacrylates. Previous studies have shown substantial cytotoxicity caused by acrylate monomers, ${ }^{[12]}$ presumably due to the high reactivity of acrylate groups with cell membrane proteins via Michael addition reactions. Additionally, products derived from the hydrolysis of polyacrylates are difficult to excrete from the human body. ${ }^{[12 \mathrm{a}]}$ Mautner et al. evaluated the structure-toxicity relationship in different classes of vinyl monomers. ${ }^{[23]}$ For the same spacer, methacrylates have significantly lower cytotoxicity than acrylate analogues. As shown in Figure 3a,b, the cytotoxicity of GelMA and PEGDA precursors were analyzed in solution with equal molar concentrations of terminal groups. After $24 \mathrm{~h}$ of incubation, GelMA solutions at all three concentrations exhibited negligible influence on cell metabolic activity (Figure 3a). By contrast, PEGDA solutions at $30 \mathrm{mM}$ and $60 \mathrm{mM}$ induced more than $90 \%$ decrease of cell viability. The diluted PEGDA solution caused a lower decrease of cell viability due to a lower concentration of acrylate groups. Furthermore, LDH results show that the cytotoxicity of GelMA solutions is negligible, whereas the cytotoxicity of PEGDA solutions is remarkably high in a 
concentration-dependent manner. To test if fabricated GelMA hydrogel microstructures are compatible with cells, we cultured human skin fibroblast cells on arrays of GelMA microstructures. Figure 3c depicts the confocal microscopy image of live-dead stained cells seeded on the helical structures. Negligible quantities of red-colored dead cells indicates excellent cell compatibility of the polymerized microstructures. Interestingly, the cells preferably attached to the helical structures (not glass) presumably due to the presence of adhesive motifs in gelatin. This indicates that 3D GelMA helical microswimmers have the ability to provide interactive surfaces that support cell adhesion, and growth.

Next, enzymatic degradability of GelMA microstructures was studied by incubating them in PBS solutions containing the enzyme collagenase (type II) at different concentrations. Figure 3d shows the influence of the collagenase concentration on degradation time. Here we define the degradation time as the time required for the microstructures to completely disappear. All the structures were fabricated with a laser power of $95 \mathrm{~mW}$ and a scanning speed of $10 \mathrm{~mm} \mathrm{~s}^{-1}$. To investigate on how size and shape of GelMA microstructures influences enzymatic degradation, both helical structures and cuboid structures were fabricated. It was found that the degradation time of helical structures was significantly shorter than the cuboid ones, because helices have much higher specific surface area (SSA) than cuboid structures. The difference is more obvious at a lower concentration of enzyme. We also observed differences in degradation times for helices with different cross-sections. Thinner swimmers, which have higher SSA, exhibited shorter degradation times. These results show that the degradation process of GelMA microstructures can be finely tuned by changing the enzyme concentration and the dimensions and shape of the swimmer. The timeresolved degradation process of a helical microswimmer in a collagenase solution $(0.1 \mathrm{mg}$ $\mathrm{mL}^{-1}$ ) is demonstrated in Figure 3e.

The enzymatic degradation of GelMA is via proteolytic cleavage of amide bonds of peptide domains in gelatin with the action of specific enzymes. In nature, mammalian cells 
have the ability to secrete various matrix metalloproteinases (MMPs) including collagenases to remodel their tissue environments if needed. The production of collagenase can be realized and enhanced by a number of biological substances in vivo and in vitro, such as soluble factors derived from human monocyte-macrophages, ${ }^{[24]}$ guinea pig lymphocytes ${ }^{[25]}$ and rabbit lymphocytes. ${ }^{[26]}$ To test if the magnetic GelMA microswimmers can be degraded by cellsecreted proteases, HaCaT cells, a keratinocyte cell line from adult human skin, were seeded on magnetic microswimmers with a density of 1760 cells per square centimeter. After incubating for 1 week, images were recorded to assess the degradation of the magnetic microswimmers (as shown in Figure S2). Then, the cells were stained by a live-dead cell imaging kit (R37601) from Thermo Fisher Scientific and imaged with a fluorescence microscope. The live-dead image is shown in Figure 3f. Figure 3g shows a reference sample of magnetic microswimmers incubated in the same cell media but without cells. The disappearance of microswimmers in Figure $3 \mathrm{f}$ (Figure S2) is attributed to cell-mediated degradation. Cells attached on the microstructures remained highly viable (>90\%) as evidenced by the live-dead staining assay (Figure 3f), indicating that both magnetic microswimmers and the residues derived from the degradation of magnetic microswimmers are not cytotoxic. To better understand the degradation process, helical and cuboid microstructures without magnetic nanoparticles were also tested using HaCaT cells. After one week of cell culture, helical structures were partially degraded, and the cuboid structures softened due to degradation (Figure $\mathbf{3 h}, \mathbf{3 i}$ and $\mathbf{3 j}$ ). These results further confirmed that the GelMA microswimmers can be degraded by cell-secreted proteases. Results regarding degradation with $3 \mathrm{~T} 3$ cells and HDFs cells (Human dermal fibroblasts) were also conducted and are shown in Figure S3 and S4. For 3T3 cells experiments, deformation of GelMA microstructures was observed after 3 days, and kept increasing with time. GelMA helical microstructures incubated with HDFs cells were dragged and deformed by cells, and degradation was observed after $36 \mathrm{~h}$. As a control, we incubated GelMA helical structures in 
the culture medium without cells to assess the stability of the structures. After 16 days, the structures preserved their helical geometry, as shown in Figure S5.
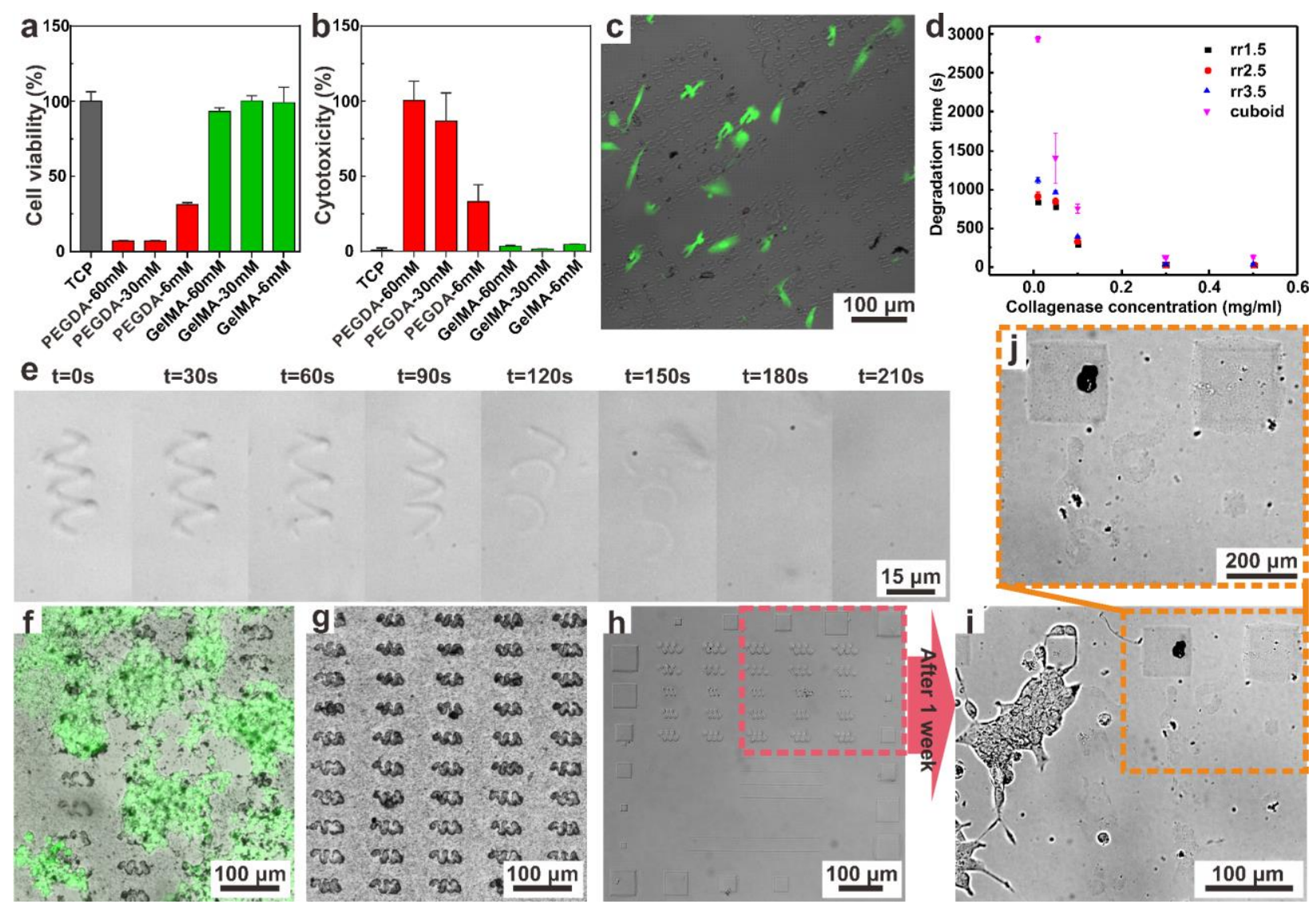

Figure 3. Biocompatibility and biodegradability of GelMA helical microswimmers. (a) Cell viability after $24 \mathrm{~h}$ of incubation with GelMA and PEGDA solutions using the MTT assay: cells cultivated on tissue culture plate were set as control (CTRL); each data set corresponds to mean \pm SD $(\mathrm{n}=4)$. (b) Cytotoxicity of GelMA and PEGDA solutions after $24 \mathrm{~h}$ of incubation using the LDH assay; each data set corresponds to mean \pm SD (n = 4). (c) A confocal microscopy image of live-dead stained human fibroblasts seeded on GelMA helices after $12 \mathrm{~h}$. The live and dead cells are green and red, respectively. Cells were highly viable and preferably attached to GelMA structures. (d) Degradation of different structures after incubation in solutions with different concentrations of collagenase. Here, the legends "rr1.5", "rr2.5", and "rr3.5" represent $30 \mu \mathrm{m}$ long microswimmers with a radius of the cylindrical cross-section of $1.5 \mu \mathrm{m}, 2.5 \mu \mathrm{m}$, and $3.5 \mu \mathrm{m}$, respectively. The legend "cuboid" represents a $50-\mu \mathrm{m} \times 50-\mu \mathrm{m} \times 10-\mu \mathrm{m}$ cuboid; each data set corresponds to mean $\pm \operatorname{SD}(\mathrm{n}=3)$. (e) 
Degradation of a GelMA helical microstructure in a collagenase solution $\left(0.1 \mathrm{mg} \mathrm{mL}^{-1}\right)$. Live-dead image after incubating HaCaT cells with magnetic GelMA microswimmers for 1 week. The live and dead cells are stained green and red, respectively. (g) Magnetic microswimmers after incubating for 1 week in cell culture medium without cells. (h) GelMA helical microstructures before cell culture. (i) Partially degraded GelMA helical microstructures after incubation with $\mathrm{HaCaT}$ cells for one week due to cell-mediated proteolysis. (j) Enlarged image of partially degraded GelMA helical microstructures after incubation with $\mathrm{HaCaT}$ cells for one week due to cell-mediated degradation.

\section{Conclusions}

In conclusion, we have developed a versatile approach to manufacture $3 \mathrm{D}$ printed proteinbased soft microrobotic systems based on noncytotoxic and biodegradable GelMA hydrogels. The procedure allows for printing microstructures with different dimensions and sizes. Because of the soft nature of GelMA, the reported helical microswimmers exhibit a unique swimming behavior under rotational magnetic fields. Compared to rigid microswimmers, which exhibit a dramatic decrease of their forward velocity above the step-out frequency, GelMA microswimmers can keep relatively high values of forward velocity as the rotating frequency increases. Cell experiments reveal that GelMA microswimmers are biodegradable, and the GelMA precursor, GelMA microswimmers, and the degradation residues of magnetic microswimmers, are not cytotoxic. Moreover, GelMA microswimmers can serve as dynamic surfaces that support cell adhesion, and growth. Due to its superior properties as a bioactive and biodegradable material and its robust photocrosslinkable features, GelMA microswimmers are promising candidates for the next generation of biomedical small-scale biodegradable robots.

\section{Experimental section}




\subsection{Synthesis of the two-photon photoinitiator}

A highly efficient water-soluble two-photon photoinitiaor (P2CK) was synthesized according to the protocol reported by the Liska group. ${ }^{[16]}$ Briefly, P2CK was prepared by aldol condensation of freshly distilled cyclopentanone $(0.1 \mathrm{~g}, 1.1 \mathrm{mmol})$ with benzaldehyde 3-[(4formyl-phenyl)-methyl-amino]-propionic acid (0.46 g, $2.2 \mathrm{mmol})$ in $2 \% \mathrm{NaOH}$ solution at $80{ }^{\circ} \mathrm{C}$ under reflux for $6 \mathrm{~h}$. After precipitation in Ethanol/HCl $(10 \mathrm{~mL})$, red-colored solids were obtained. After washing with cold Methanol, the products were dried under vacuum and used without further purification.

\subsection{Synthesis of the hydrogel precursor}

The GelMA precursor was derived from Type B gelatin isolated from bovine skin (Sigma, bloom strength 225). This material has an isoelectric point of 5.0. The synthesis of GelMA was performed as described by Van Den Bulcke et al. ${ }^{[27]}$ In brief, the lysine units of gelatin were reacted with methacrylic anhydride in PBS for $12 \mathrm{~h}$. The crude products were purified by sequential dialysis in $\mathrm{NaHCO}_{3}(200 \mathrm{mM})$ for $24 \mathrm{~h}$ and in distilled water for $24 \mathrm{~h}$ at $40{ }^{\circ} \mathrm{C}$ with 4 times of water change per day. After lyophilization, white solids were obtained in $79 \%$ yield. ${ }^{1} \mathrm{H}-\mathrm{NMR}$ analysis shows that a degree of substitution of $70 \%$ was obtained (as shown in Figure S6).

\subsection{Photorheology}

Prior to two-photon microfabrication, photoreactivity of GelMA precursors was measured on a Anton-Paar MCR301 photorheometer as described in a recent work. ${ }^{[28]}$ In brief, $100 \mu \mathrm{L}$ of $20 \%$ GelMA solutions in PBS containing $0.5 \%$ Irgacure 2959 (chemical structure shown in Scheme 2) was pipetted to the quartz plate. After positioning the upper plate to a gap thickness of $150 \mu \mathrm{m}$, a time-sweep measurement was initiated at a fixed frequency of $1 \mathrm{~Hz}$ and strain of $0.5 \%$. After $60 \mathrm{~s}$, a UV-365 lamp (Omnicure S1000, $20 \mathrm{~mW} \cdot \mathrm{cm}^{-2}$ ) was shuttled on to induce radical photopolymerization. The evolution of shear storage modulus ( $\left.\mathrm{G}^{\prime}\right)$ of the samples was monitored for $3 \mathrm{~min}$. In a control experiment, $2 \%$ iron oxide nanoparticles were 
included into GelMA solutions to screen its effects on photocrosslinking and final gel modulus.

\subsection{Hydrogel microfabrication and characterization}

The Nanoscribe system (photonic Professional GT) was used to fabricate the GelMA microstructures. A femtosecond laser of $780 \mathrm{~nm}$ and $80 \mathrm{MHz}$, and a $63 \times$ objective was used in the system. GelMA (200 $\left.\mathrm{mg} \mathrm{mL}^{-1}\right)$ and the photoinitiator P2CK $\left(1.1 \mathrm{mg} \mathrm{mL}^{-1}\right)$ were dissolved in PBS at $45{ }^{\circ} \mathrm{C}$ to prepare the precursor solution. The precursor solution was deposited on the center of glass slide where a GelMA photoresist film developed upon cooling. Then, a near-infrared femtosecond laser scanned the photoresist to trigger the polymerization. The photoresist was then developed in water at $45{ }^{\circ} \mathrm{C}$ for $0.5 \mathrm{~h}$. For magnetic actuation, GelMA microstructures were decorated with $\mathrm{Fe}_{3} \mathrm{O}_{4}$ nanoparticles by incubating them in a water suspension of $\mathrm{Fe}_{3} \mathrm{O}_{4}\left(10 \mathrm{mg} \mathrm{mL}^{-1}, 1 \%\right.$ PVP coated $)$ for different times. Afterwards, the sample was rinsed with DI water for several times to remove non-adsorbed nanoparticles. $\mathrm{Fe}_{3} \mathrm{O}_{4}$ magnetic nanoparticles were purchased from Nanostructured and Amorphous (USA).

Optical images were taken using an integrated inverted optical microscope (Olympus IX-81). The sample for the cryo-SEM and EDX was pretreated in the following way. First, GelMA helical microswimmers were prepared on a sapphire disc and covered by water. Then the sample was plunged into a liquid ethane/propane mixture (37\% Ethane), and loaded into BAF-060 specimen table (Leica, Vienna) at $-120{ }^{\circ} \mathrm{C}$. Hereafter, partial freeze-drying (from $120{ }^{\circ} \mathrm{C}$ to $-95{ }^{\circ} \mathrm{C}$ with $60{ }^{\circ} \mathrm{C} / \mathrm{h}$ ramp, $0.5 \mathrm{~h}$ at $-95{ }^{\circ} \mathrm{C}$, back to $-120{ }^{\circ} \mathrm{C}$ ) was carried out to partially remove ice layer that covered the microswimmers. Afterwards, the sample was coated with tungsten $(1 \mathrm{~nm})$ at $45^{\circ}$ angle at $-120^{\circ} \mathrm{C}$ and transferred into the microscope via VCT100 cryo transfer system (Leica, Vienna) under vacuum and cryogenic conditions. The cryo-SEM and EDX were taken using Leo-1530 scanning electron microscope (Zeiss, Oberkochen) at $-120^{\circ} \mathrm{C}$. 


\subsection{Swimming performance}

A Helmholtz setup consisting of three pairs of orthogonal coils were used to generate rotating magnetic fields for the swimming test. The sample holder with a helical microswimmer sample inside was filled with a biologically relevant solution $\left(38 \mathrm{mg} \mathrm{mL}^{-1}\right.$ bovine serum albumin and $0.9 \mathrm{mg} \mathrm{mL}^{-1}$ glucose in PBS, $\mathrm{pH}=7.4$ ), and placed at the center of the Helmholtz setup to ensure the uniformity of the magnetic field. A microprobe (T-4-22, GGB Industries, INC.) was used for the release and transportation of microswimmers. A rotating magnetic field of $8 \mathrm{mT}$ was applied to perform the swimming test of the fabricated magnetic helical microswimmers. Values of velocities result from averaging 4 measurements and error bars represent the standard deviations.

\subsection{Cytotoxicity assay}

Cytotoxicity of GelMA macromer solutions was evaluated via MTT and LDH assay in comparison with PEGDA solutions with equal molar concentration of functional groups: 6 $\mathrm{mM}, 30 \mathrm{mM}, 60 \mathrm{mM}$. For both assays, C2C12 myoblasts were cultured in Dulbecco's Modified Eagles Medium (DMEM) supplemented with $10 \%$ fetal calf serum (FCS), $1 \%$ LGlutamine, $1 \%$ Penicillin/Streptomycin (Sigma-Aldrich). Macromer solutions with three concentrations were dissolved in DMEM medium. Cells were then seeded in a 96-well plate at a density of $6 \times 10^{3}$ cells per well in culture medium $(200 \mu \mathrm{L})$. After $24 \mathrm{~h}$ of incubation at $37{ }^{\circ} \mathrm{C}$ and $5 \% \mathrm{CO}_{2}$, the respective macromer solutions $(100 \mu \mathrm{L})$ were added to the cells in triplicates. After $24 \mathrm{~h}$ incubation, cells were washed twice with sterile PBS before the addition of thiazolyl blue tetrazolium bromide (MTT) working solution (100 $\mu \mathrm{L}, 5 \mathrm{mg} \mathrm{mL}^{-1}$ in PBS). After $2 \mathrm{~h}$ incubation, the liquid was discarded and DMSO (100 $\mu \mathrm{l})$ was added to dissolve the formazan crystals. Finally, the absorbance was measured at $540 \mathrm{~nm}$ using a microplate reader. For LDH assay, a similar procedure was performed but without adding the cell lysis buffer after $24 \mathrm{~h}$ of incubation to create positive control (100\% dead cells). After $30 \mathrm{~min}$, supernatants $(50 \mu \mathrm{L})$ from each well were transferred to a new 96-well plate and added with 
reaction buffer $(50 \mu \mathrm{L})$. After $30 \mathrm{~min}$, stop buffer $(50 \mu \mathrm{L})$ was added to stop the colorization. Finally, the absorbance was measured at $540 \mathrm{~nm}$ using a microplate reader.

\subsection{Cell Seeding and live-dead assay of GelMA helical microstructures}

HDFs in suspension $\left(10 \times 10^{3}\right.$ cells $\left.\mathrm{cm}^{-2}\right)$ were seeded on helical structures of GelMA and cultured in serum-free DMEM medium for up to $96 \mathrm{~h}$ to monitor cell-material interactions. For confocal imaging, samples were washed with PBS after $12 \mathrm{~h}$, and stained with live (calcein-AM) or dead (ethidium homodimer-1) dyes for $30 \mathrm{~min}$. The cells were imaged at 488/561 nm with an integrated phase-contrast transmission camera for visualization of GelMA microstructures.

\subsection{Degradation of GelMA microstructures with enzyme solutions}

$50-\mu \mathrm{m} \times 50-\mu \mathrm{m} \times 10-\mu \mathrm{m}$ cuboid structures and helical microswimmers with a length of $30 \mu \mathrm{m}$ and different radius of cylindrical cross-section $(1.5 \mu \mathrm{m}, 2.5 \mu \mathrm{m}$ and $3.5 \mu \mathrm{m})$ were fabricated for evaluating their degradation in solutions containing collagenase (Sigma-Aldrich) at different concentrations $\left(0.01 \mathrm{mg} \mathrm{mL}^{-1}, 0.05 \mathrm{mg} \mathrm{mL}^{-1}, 0.1 \mathrm{mg} \mathrm{mL}^{-1}, 0.3 \mathrm{mg} \mathrm{mL}^{-1}\right.$, and $0.5 \mathrm{mg}$ $\left.\mathrm{mL}^{-1}\right)$.

\subsection{Degradation of GelMA helical microswimmers by cell-secreted proteases}

Several cell types (HaCaT, 3T3, and HDFs) were screened for their interactions with GelMA microswimmers. Samples were washed with $70 \%$ ethanol in DI water for 3 times for sterilization and then washed with sterile PBS to remove ethanol residues. After diluting cells to the target density, cells in suspension (1700-2000 cells $\left.\mathrm{cm}^{-2}\right)$ were seeded on GelMA helical microswimmers and cultured in a medium of DMEM with $10 \%$ Fetal Bovine Serum (FBS) and $1 \%$ Antibiotic-Antimucotic. After culturing for a certain time in an incubator with $5 \% \mathrm{CO}_{2}$ and a temperature of $37^{\circ} \mathrm{C}$, the sample was taken out to take pictures or kept for the next step. Live-dead cell imaging kit (R37601) from Thermo Fisher Scientific was used for the live-dead staining for $15 \mathrm{~min}$ after the degradation experiment of magnetic 
microswimmers with HaCaT cells. The cells were imaged at 488/570 nm for visualization.

The control groups were done in the same way, but without any cell.

\section{Supporting information}

Supporting Information is available from the Wiley Online Library or from the author.

\section{Acknowledgements}

The work has been partially funded by the European Research Council (ERC) Advanced Grant "Soft Micro Robotics” (SOMBOT) under the European Union's Horizon 2020 research and innovation programme (grant agreement No. 743217). X. W. acknowledges financial support from China Scholarship Council (No: 201504910817). X. Q. was supported by a EU Marie-Curie Postdoctoral Fellowship (COFUND 267161). T. H. acknowledges financial support from National Natural Science Foundation of China (No.11702003). The authors thank Lucas Falk for the Cryo-SEM and EDX, and the FIRST lab and ScopeM of ETH Zurich for technical support.

\section{References}

[1] a) B. J. Nelson, I. K. Kaliakatsos, J. J. Abbott, Annu. Rev. Biomed. Eng. 2010, 12, 55; b) S. Palagi, P. Fischer, Nat. Rev. Mater. 2018, 3, 113; c) M. Sitti, Nat. Rev. Mater. 2018, 3, 74; d) W. Hu, G. Z. Lum, M. Mastrangeli, M. Sitti, Nature 2018, 554, 81.

[2] a) F. Qiu, R. Mhanna, L. Zhang, Y. Ding, S. Fujita, B. J. Nelson, Sens. Actuators, B 2014, 196, 676; b) M. Hoop, F. Mushtaq, C. Hurter, X.-Z. Chen, B. Nelson, S. Pané, Nanoscale 2016, 8, 12723; c) X. Z. Chen, M. Hoop, N. Shamsudhin, T. Huang, B. Özkale, Q. Li, E. Siringil, F. Mushtaq, L. Di Tizio, B. J. Nelson, Adv. Mater. 2017, 29, 1605458; d) F. Qiu, S. Fujita, R. Mhanna, L. Zhang, B. R. Simona, B. J. Nelson, Adv Funct Mater 2015, 25, 1666; e) X. Lin, Z. Wu, Y. Wu, M. Xuan, Q. He, Adv. Mater. 2016, 28, 1060; f) J. Shao, M. Xuan, H. Zhang, X. Lin, Z. Wu, Q. He, Angew. Chem. 2017, 129, 13115.

[3] S. Guo, Q. Pan, M. B. Khamesee, Microsyst. Technol. 2008, 14, 307.

[4] A. Chałupniak, E. Morales-Narváez, A. Merkoçi, Adv. Drug Delivery Rev. 2015, 95, 104.

[5] A. Servant, F. Qiu, M. Mazza, K. Kostarelos, B. J. Nelson, Adv. Mater. 2015, 27, 2981.

[6] X. Wang, C. Hu, L. Schurz, C. De Marco, X. Chen, S. Pané, B. J. Nelson, ACS Nano 2018, $12,6210$.

[7] a) S. Palagi, A. G. Mark, S. Y. Reigh, K. Melde, T. Qiu, H. Zeng, C. Parmeggiani, D. Martella, A. Sanchez-Castillo, N. Kapernaum, Nat. Mater. 2016, 15, 647; b) H.-W. Huang, M. S. Sakar, A. J. Petruska, S. Pané, B. J. Nelson, Nat. Commun. 2016, 7 , 12263; c) H.-W. Huang, A. J. Petruska, B. J. Nelson, Homeland Defense \& Security Information Analysis Center Journal 2017, 25; d) H.-W. Huang, Q. Chao, M. S. Sakar, B. J. Nelson, IEEE Robotics and Automation Letters 2017, 2, 727; e) N. Hu, M. Sun, 
X. Lin, C. Gao, B. Zhang, C. Zheng, H. Xie, Q. He, Adv. Funct. Mater. 2018, 28, 1705684.

[8] a) C. Peters, M. Hoop, S. Pané, B. J. Nelson, C. Hierold, Adv. Mater. 2016, 28, 533; b) H. Ceylan, I. C. Yasa, M. Sitti, Adv. Mater. 2017, 29, 1605072.

[9] a) J. Li, B. E.-F. de Ávila, W. Gao, L. Zhang, J. Wang, Sci. Robot. 2017, 2, eaam6431; b) Z. Wu, X. Lin, X. Zou, J. Sun, Q. He, ACS Appl. Mater. Interfaces 2014, 7, 250; c) J.-y. Kim, S. Jeon, J. Lee, S. Lee, J. Lee, B. O. Jeon, J. E. Jang, H. Choi, Sens.

Actuators, $B$ 2018, 266, 276; d) Y. Tu, F. Peng, A. A. André, Y. Men, M. Srinivas, D. A. Wilson, ACS Nano 2017, 11, 1957.

[10] C. Chen, E. Karshalev, J. Li, F. Soto, R. Castillo, I. Campos, F. Mou, J. Guan, J. Wang, ACS Nano 2016, 10, 10389.

[11] Y. Tu, F. Peng, P. B. White, D. A. Wilson, Angew. Chem. 2017, 56, 7620.

[12] a) C. Heller, M. Schwentenwein, G. Russmueller, F. Varga, J. Stampfl, R. Liska, J. Polym. Sci., Part A: Polym. Chem. 2009, 47, 6941; b) X.-H. Qin, A. Ovsianikov, J. Stampfl, R. Liska, BioNanoMaterials 2014, 15, 49.

[13] P. Lu, K. Takai, V. M. Weaver, Z. Werb, Cold Spring Harbor Perspect. Biol. 2011, 3, a005058.

[14] D. Loessner, C. Meinert, E. Kaemmerer, L. C. Martine, K. Yue, P. A. Levett, T. J. Klein, F. P. Melchels, A. Khademhosseini, D. W. Hutmacher, Nat. Protoc. 2016, 11, 727.

[15] K. E. Peyer, A. W. Mahoney, L. Zhang, J. J. Abbott, B. J. Nelson, in Microbiorobotics, Elsevier 2012, p. 165.

[16] Z. Li, J. Torgersen, A. Ajami, S. Mühleder, X. Qin, W. Husinsky, W. Holnthoner, A. Ovsianikov, J. Stampfl, R. Liska, RSC Adv. 2013, 3, 15939.

[17] X.-H. Qin, P. Gruber, M. Markovic, B. Plochberger, E. Klotzsch, J. Stampfl, A. Ovsianikov, R. Liska, Polym. Chem. 2014, 5, 6523.

[18] a) J. Van Hoorick, P. Gruber, M. Markovic, M. Tromayer, J. r. Van Erps, H. Thienpont, R. Liska, A. Ovsianikov, P. Dubruel, S. Van Vlierberghe,

Biomacromolecules 2017, 18, 3260; b) L. Brigo, A. Urciuolo, S. Giulitti, G. Della Giustina, M. Tromayer, R. Liska, N. Elvassore, G. Brusatin, Acta Biomater. 2017, 55, 373.

[19] a) Y. Kamiyama, J. Israelachvili, Macromolecules 1992, 25, 5081; b) A. Kamyshny, O. Toledano, S. Magdassi, Colloids and Surfaces B: Biointerfaces 1999, 13, 187; c) I. Mironyuk, V. Gun'ko, V. Turov, V. Zarko, R. Leboda, J. Skubiszewska-Zięba, Colloids and Surfaces A: Physicochemical and Engineering Aspects 2001, 180, 87; d) B. Gaihre, M. S. Khil, D. R. Lee, H. Y. Kim, International Journal of Pharmaceutics 2009, 365, 180.

[20] T. H. C. Salles, C. B. Lombello, M. A. d'Ávila, Materials Research 2015, 18, 509.

[21] F. Qiu, B. J. Nelson, Engineering 2015, 1, 021.

[22] a) B. Ten Hagen, F. Kümmel, R. Wittkowski, D. Takagi, H. Löwen, C. Bechinger, Nat. Commun. 2014, 5, 4829; b) S. Tottori, L. Zhang, F. Qiu, K. K. Krawczyk, A. Franco

- Obregón, B. J. Nelson, Adv. Mater. 2012, 24, 811.

[23] A. Mautner, X. Qin, H. Wutzel, S. C. Ligon, B. Kapeller, D. Moser, G. Russmueller, J. Stampfl, R. Liska, J. Polym. Sci., Part A: Polym. Chem. 2013, 51, 203.

[24] a) J. Dayer, D. Robinson, S. Krane, J. Exp. Med. 1977, 145, 1399; b) J. Dayer, J. Breard, L. Chess, S. Krane, J. Clin. Investig. 1979, 64, 1386.

[25] L. M. Wahl, S. M. Wahl, S. E. Mergenhagen, G. R. Martin, Science 1975, 187, 261.

[26] D. A. Newsome, J. Gross, Cell 1979, 16, 895.

[27] A. I. Van Den Bulcke, B. Bogdanov, N. De Rooze, E. H. Schacht, M. Cornelissen, H. Berghmans, Biomacromolecules 2000, 1, 31 . 
[28] X. H. Qin, X. Wang, M. Rottmar, B. J. Nelson, K. Maniura - Weber, Adv. Mater. 2018, 30, 1705564. 
Biodegradable soft helical microswimmers are successfully developed based on two-photon photopolymerization of a gelatin derivative, GelMA. By decorating the surface of helical microstructures with magnetic nanoparticles, microswimmers which can be manipulated by magnetic field are fully developed. Because of the proteolytic cleavage of peptide domains in gelatin, microswimmers made of GelMA can be fully degraded by cell-secreted proteases.

Keywords: biodegradable hydrogel, two-photon polymerization, soft helical microswimmers, magnetic manipulation

X. Wang, Dr. X.-H. Qin, Dr. C. Hu, A. Terzopoulou, Dr. X.-Z. Chen, Dr. T.-Y. Huang, Prof. K. Maniura-Weber, Dr. S. Pané, Prof. B. J. Nelson

3D Printed Enzymatically Biodegradable Soft Helical Microswimmers

ToC figure

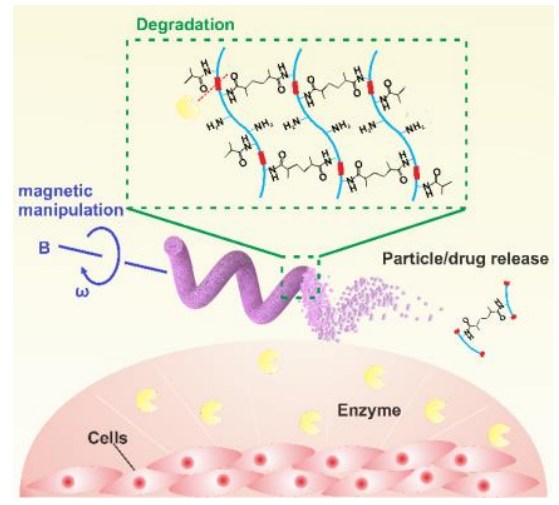

